\title{
Research Education, Distance Learning, and the COVID-19 Era
}

\author{
Marsal Sanches ${ }^{1}$ (C)
}

Received: 16 June 2020 / Accepted: 28 October 2020 / Published online: 17 November 2020

(C) Academic Psychiatry 2020

Over the past two years, I have had the pleasure of running regular didactic sessions with our psychiatry research track residents. Sometimes, those sessions are held in a traditional journal club format, with one resident responsible for the presentation of the article, followed by an open discussion on the content of the paper and, in particular, its methodological aspects. Other times, we do a around-the-table discussion of a specific paper, usually a reflective piece on the directions psychiatric research seems to be heading towards. That was the case last week, when we discussed a review article focusing on the limitations and perspectives related to different approaches we can use to conceptualize the psychiatric diagnosis [1]. So far, nothing new. Except for the fact that, due to the COVID-19 pandemic and the recommended social distancing measures, our department, as many others, had to convert most of our residency didactic activities to distance learning. Instead of meeting in person for a literal around-the-table session, we met online, using one of the several available video conference platforms.

I am not particularly tech savvy but did not think it would be a problem. By now, I have performed telehealth appointments extensively and have participated in different video conferences and virtual meetings, and things usually seem to go fairly well. Every now and then there were issues with someone forgetting to mute their microphone while another person is speaking, as well as a poor internet connection that would cause some of the participants to have their images frozen. Nothing that could not be easily fixable.

This time, however, it was different. As I started to speak, I noticed that virtually everyone had not only their microphones but also their videos in "off" mode. I could not see the faces of those who were present in the session and, worse, could not say who was paying attention and who was not and even whether or not they were hearing what I was saying. Some

Marsal Sanches

Marsal.Sanches@uth.tmc.edu

1 The University of Texas Health Science Center at Houston (UTHealth), Houston, TX, USA people had called from their phones instead of joining the sessions from their computers, and that added an extra problem: in addition to not being able to see those participants' faces, it was not even possible to know for sure who they were, as they were identified just by their respective phone numbers and not by their names. As I tried to ask questions to the audience about the topic we were discussing, there were usually long gaps, and at times some participants would make comments through the chat feature of the platform we were utilizing, instead of speaking up. After a while, I no longer could resist and asked the residents why they were keeping their videos off. Was it because the connection was poor? I heard the most curious answers, such as "I am actually driving home," "my house is too messy," "we are just shy," and, most importantly, "that's how we have been doing during all other lectures."

I am quite used to giving lectures and really enjoy it. Still, as the facilitator of a discussion, I always rely on people's facial cues and body posture to assess whether or not I am being successful in engaging them and to make adjustments and further clarifications to what I am saying. I like the interactions and sometimes the arguments that result from a particularly controversial topic, especially when it comes to research education. Similarly to what happens with psychotherapy training, nobody learns research by mutely watching someone talking about it. It is not difficult to find good online resources, including videos on a variety of topics. You can surely learn about research by watching them. However, can you learn how to do research if you limit your learning to the unilateral watching of a video? Prior to the onset of the COVID-19 pandemic, many medical schools around the country already offered their students the opportunity of watching live-streamed lectures instead of physically attending them [2]. That possibility may be regarded as an attractive alternative to getting dressed up, driving to the site where the lecture was being given, struggling to find a parking spot, and, finally, spending two hours in a crowded room watching a lecturer talk non-stop and monotonously displaying slide after slide. If that is the case, I agree: streaming the lectures makes much more sense. 
Nevertheless, the unique differential offered by going to a university instead of staying home and reading books or watching videos has to do much more with the possibility of exchanging impressions and experiences than with being provided with knowledge. One of my medical school professors, back in the early 1990s, used to joke about how we "learn a lot by rubbing our brains against each other." If the process is unilateral, there is no actual "brain rubbing." That is not something new, and educators have been, for decades, discussing alternative models that could potentially replace the traditional lecture approach. Problem-based learning (PBL), flipped classroom, and many other innovative approaches all seem to have their strengths and limitations, and it will be very interesting to assess how they will navigate the COVID-19 era. Is it feasible to properly conduct a PBL session online, if the participants' videos are off and are not able to talk among themselves in smaller groups? What would be the role of the facilitator in this new reality?

I do not have the answers to those questions, and I am not at all against distance learning, specially in the current scenario. I only think we (and, by we, I mean faculty members, residents, and students) should have an open discussion about it, in order to achieve a certain level of compromise as for how to conduct an online teaching session. For example, the simple habit of sharing with the residents and students, in advance, a list of expectations concerning an upcoming lecture (e.g., recommending the audience to be prepared to keep their cameras on, to mute their microphones when not speaking, and, when possible, to minimize environmental distractors) might help the speaker and the audience establish a common ground for the didactic session, making the experience in question much more productive and enjoyable. Furthermore, members of the audience might feel particularly uncomfortable with interrupting the speaker to ask a question during an online teaching session, and planning for intermittent, brief pauses in the flow of the lecture, during which the spectators should be asked if there are any questions or comments, is likely recommendable. Lecturers might have different levels of comfort with the use of the chat window, and one possibility is assigning a co-presenter (or a volunteer among the audience members) to monitor the questions and comments posted through chat and read or synthetize them during the pauses mentioned above. I recently tried that, with good results. Finally, there are several options in terms of audience response applications, which facilitate live interactions with the listeners and may be particularly useful during online teaching involving large audiences.

I recently heard a historian stating that pandemics have a catalyzing effect on emerging changes in society, some of which might already had been rehearsed but had been facing some resistance. If that is true, distance learning is here to stay, and we will have to deal with it. And make the most out of it.

\section{Compliance with Ethical Standards}

Conflict of Interest The author states that there is no conflict of interest.

\section{References}

1. Borsboom D. Psychometric perspectives on diagnostic systems. J Clin Psychol. 2008;64:1089-108.

2. Sandhu A, Fliker A, Leitao D, Jones J, Gooi A. Adding livestreaming to recorded lectures in a non-distributed pre-clerkship medical education model. Stud Health Technol Inform. 2017;234: 292-7.

Publisher's Note Springer Nature remains neutral with regard to jurisdictional claims in published maps and institutional affiliations. 